\title{
Programa de tutoria em Bioquímica na Universidade Federal de Viçosa
}

\section{Tutoring Program in Biochemistry at the Federal University of Viçosa}

\author{
Bruna M.Araújo ${ }^{1,2 *}$, Éverton de A.A. Barbosa ${ }^{1,2,}$, Sandra G. Gava ${ }^{1,2,}$, Zaira B. Hoffmam ${ }^{1,2}$,'Tânus Henrique A. \\ Pereira $^{1,2}$, Flávio A.Silva ${ }^{1,3}$, Nayara G. Tessarollo ${ }^{1,2}$, Andréa O.B.Ribon ${ }^{1,2^{*}}$ \\ *e-mail: abribon@ufv.br \\ ${ }^{1}$ Programa de Apoio às Ciências Básicas, Universidade Federal de Viçosa \\ ${ }^{2}$ Departamento de Bioquímica e Biologia Molecular, Universidade Federal de Viçosa. \\ ${ }^{3}$ Departamento de Química, Universidade Federal de Viçosa.
}

\section{Resumo}

Este estudo descreve o Programa de Tutoria em Bioquímica (TutBQI) da Universidade Federal de Viçosa, uma atividade extracurricular proposta para complementar as aulas expositivas tradicionais na disciplina de Bioquímica Fundamental (BQI 100). Dez anos após a implantação do TutBQI, são relatados os impactos positivos do programa sobre o índice de reprovação e o rendimento médio dos estudantes da BQI100. São também apresentadas as limitações do programa.

Palavras-chave: ensino de bioquímica, aprendizagem em pequenos grupos, desempenho acadêmico.

\begin{abstract}
This study describes the Tutoring Program in Biochemistry (TutBQI) at the Federal University of Viçosa, an extracurricular activity designed to complement the traditional lectures in the Fundamentals of Biochemistry course (BQI 100). Ten years after the TutBQI implementation, we reported its positive impacts on the student's performance verified by the survey of the failure rates and average grades in BQI100. Limitations of the program are also presented.
\end{abstract}

Keywords: biochemistry teaching; small-group learning; student's performance. 


\section{Introdução}

Um contingente universitário cada vez mais numeroso e heterogêneo formado por estudantes com diferentes níveis de conhecimento prévio, tem sido observado nas instituições de ensino superior (IES) no Brasil. Um dos motivos para tal cenário é a vertiginosa expansão do ensino superior no país a partir do final da década de noventa, como comprova o Censo de Educação Superior (Figura 1) [1]. A Universidade Federal de Viçosa apresentou, entre 1998 e 2010, um crescimento de $80 \%$ no número de cursos oferecidos e de $93 \%$ no número de ingressos na graduação presencial (Figura 2). Essa instituição com comprovada tradição no ensino e na pesquisa na área de Ciências Agrárias possui cerca de 11.000 estudantes matriculados em seus 44 cursos de graduação.

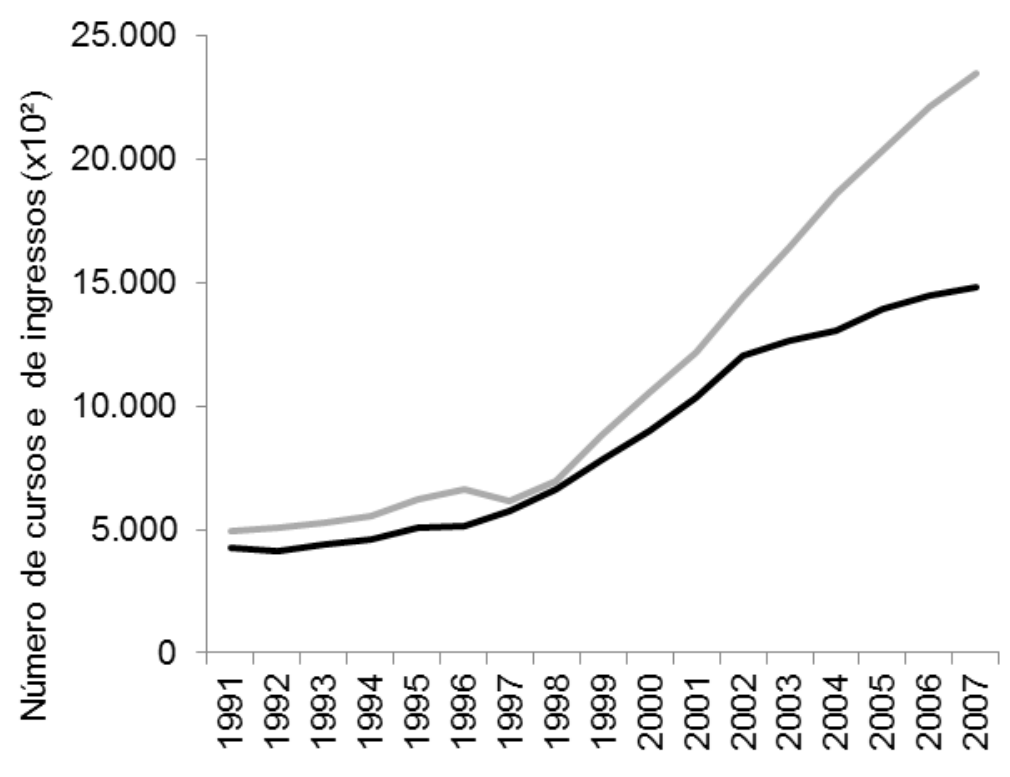

Ano

Figura 1. Evolução no número de cursos (linha cinza) e no número de ingressos (x10²) (linha preta) oferecidos nas IES, entre 1991 e 2007.

Analisando a relação entre o conhecimento prévio e o desempenho acadêmico, verificou-se, na UFV, que os cursos que selecionavam candidatos com menor pontuação no processo seletivo, apresentavam os maiores índices de reprovação em disciplinas do ciclo básico [2].

Essa análise sugeriu que a deficiência no conhecimento prévio vinha sendo responsável, pelo menos em parte, pelo aumento do tempo de permanência na universidade, pela deficiência no aproveitamento das disciplinas profissionalizantes e pela evasão escolar [2]. Diante dessa complexa realidade e considerando a necessidade de se 
assegurar a qualidade acadêmica, tornou-se imprescindível a adoção de uma política institucional que promovesse a adaptação do estudante ao ambiente universitário. Foi com esse objetivo que a UFV instituiu no ano de 2000 o Programa de Apoio Didático às Disciplinas Básicas (PAB) ou Programa de Tutoria, um programa de acompanhamento acadêmico destinado aos estudantes com deficiência no conhecimento prévio.

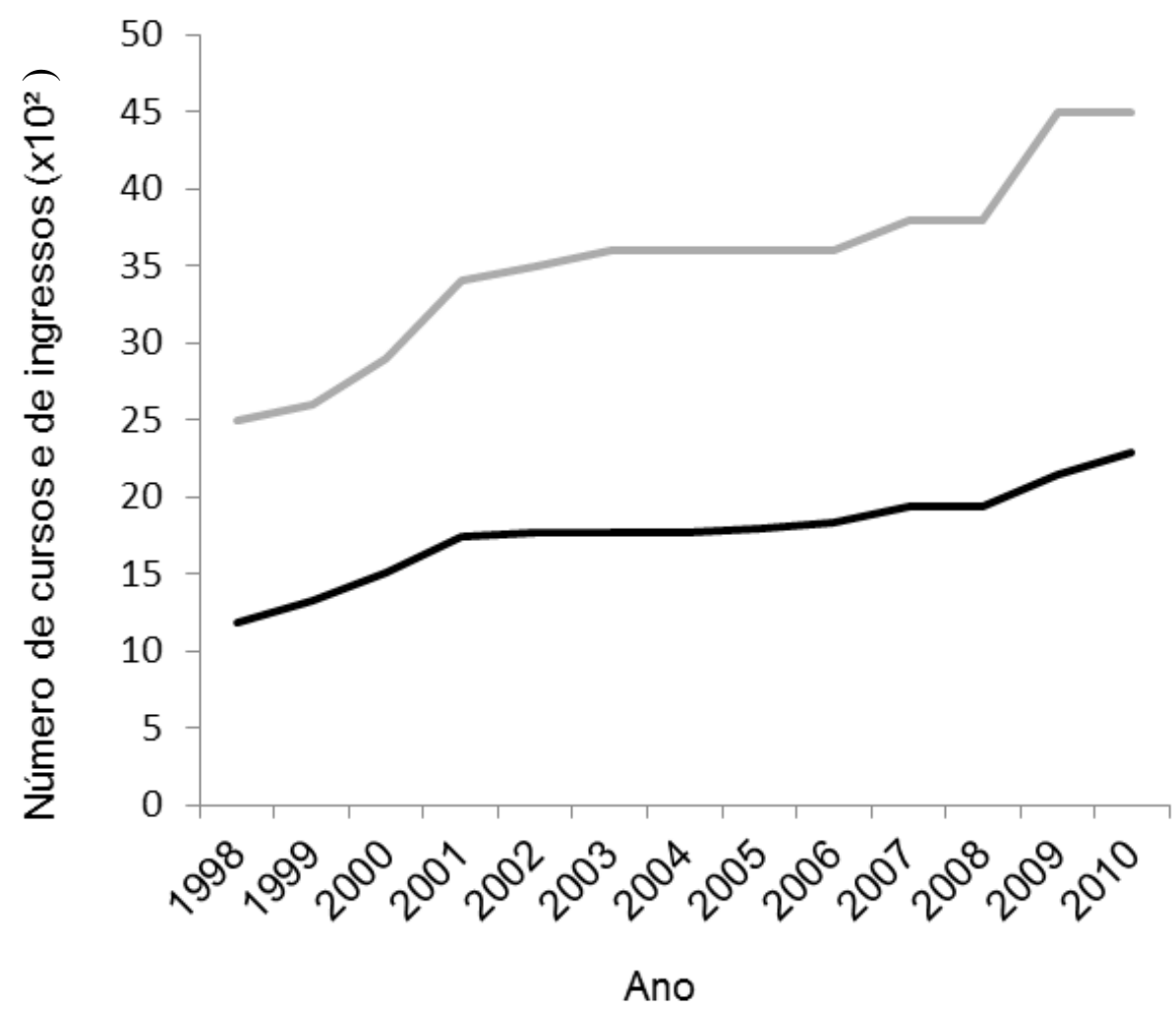

Figura 2. Evolução no número de cursos (linha cinza) e no número de ingressos $\left(\times 10^{2}\right)$ (linha preta) na graduação presencial, na UFV, entre 1998 e 2010.

O PAB oferece suporte às disciplinas que comportam um grande número de estudantes e que tendem a apresentar altos índices de reprovação ou evasão. Inicialmente ele atendia às áreas de Matemática, Física, Química, Biologia, Português e Química. Em 2001, o programa foi estendido à Bioquímica, devido aos altos índices de reprovação dos estudantes em Bioquímica Fundamental (BQI 100). Essa disciplina é oferecida semestralmente a uma média de 300 estudantes que cursam Agronomia, Engenharia Agrícola e Ambiental, Engenharia Florestal, Ciência e Tecnologia de Laticínios, Engenharia Ambiental, Engenharia de Alimentos, Engenharia Química, Física (licenciatura), Química e Economia Doméstica. Cada professor adota as aulas expositivas tradicionais com duração de 90 minutos e é responsável por uma turma com aproximadamente 80 estudantes, que podem ou não ser do mesmo curso. 


\section{Programa de tutoria em Bioquímica}

O Programa de Tutoria em Bioquímica (TutBQI) é uma atividade extracurricular oferecida semestralmente para cerca de 150 estudantes matriculados na BQI100 com o propósito de auxiliar e motivar os estudantes no seu processo de ensino-aprendizagem, corrigindo desníveis de conhecimento prévio e melhorando o desempenho acadêmico. Os estudantes são automaticamente matriculados no programa de acordo com os seguintes critérios: desempenho em Química Orgânica inferior a $70 \%$ no processo seletivo para ingresso na graduação, já que essa disciplina é pré-requisito para a BQI100, e reprovação em BQI 100. Outros estudantes podem ser admitidos desde que haja vagas remanescentes.

\section{Estrutura organizacional}

Assim como os outros programas de tutoria da UFV, a estrutura organizacional do TutBQI é composta por coordenadora geral, coordenadora setorial, tutores e profissionais de apoio. A coordenadora geral promove a comunicação entre a Pró-Reitoria de Ensino e o PAB, gerenciando todas as disciplinas oferecidas pelo programa, defendendo os interesses e necessidades do PAB e assegurando o seu enquadramento dentro das normas da universidade. A coordenadora setorial da área de Bioquímica é uma professora do Departamento de Bioquímica e Biologia Molecular nomeada para organizar as atividades que serão desenvolvidas e coordenar o TutBQI. Reúne-se quinzenalmente com os tutores e periodicamente com a coordenação geral, além de manter contato com a coordenação da BQI100. Os tutores nível I são estudantes regularmente matriculados nos cursos de graduação da UFV, selecionados por meio de provas e que conduzem as sessões de tutoria. Somente pode ser tutor o estudante que cursou e obteve um rendimento acima de $75 \%$ em alguma disciplina de Bioquímica oferecida na UFV. O tutor nível II é um estudante de pós-graduação que oferece suporte ao programa, confeccionando material didático e avaliando semestralmente o nível de satisfação dos estudantes por meio de questionários. Os profissionais de apoio compreendem o secretário administrativo e as atendentes que interagem com os estudantes, tutores e coordenadores. 


\section{Material didático}

Como material didático de apoio ao Programa TutBQI, inicialmente foram desenvolvidos dois cadernos didáticos: Tutoria em Bioquímica - Biomoléculas [3] e Tutoria em Bioquímica - Metabolismo Celular [4]. Esses cadernos foram divididos em capítulos, cada qual contendo uma breve introdução do assunto a ser estudado, um roteiro de estudo e uma lista de exercícios. Atualmente, além desses cadernos impressos, são disponibilizados online uma série de exercícios adicionais, figuras e tabelas extraídos de livros-texto de Bioquímica e vídeos interativos.

\section{Sessões de tutoria}

As sessões de tutoria são sempre relativas ao conteúdo previamente ministrado em sala de aula, de forma que quem direciona o conteúdo trabalhado são sempre os professores da BQI100. Reuniões entre o coordenador setorial e os tutores, além do conhecimento prévio dos tópicos da disciplina, auxiliam na definição de pontos essenciais que devem ser abordados nas sessões de tutoria para o entendimento de cada capítulo lecionado na BQI100.

As sessões de tutoria, com duração de 100 minutos, são realizadas semanalmente em grupos de seis estudantes, selecionados aleatoriamente, de acordo com seus horários de aulas. Esse pequeno grupo é essencial para que o tutor conheça as limitações de cada estudante e trabalhe sempre no sentido de estabelecer uma relação de confiança com os mesmos. Durante as sessões, os tutores utilizam o quadro-negro e o material didático impresso para revisar o conteúdo previamente ministrado em sala de aula e reforçar os conceitos mal assimilados pelos estudantes. Sempre que possível, os tutores encorajam a participação ativa dos estudantes, convidando-os ao quadro-negro. O tempo restante é dedicado à resolução de problemas com auxílio dos tutores.

A frequência nas sessões de tutoria define o conceito do estudante como satisfatório $(\mathrm{S})$ ou não-satisfatório $(\mathrm{N})$. Esse último é dado para aqueles estudantes que tiverem falta superior a $25 \%$. O conceito $\mathrm{S}$ ou $\mathrm{N}$ é, então, registrado no histórico escolar do estudante. $\mathrm{O}$ estudante que obtiver o conceito $\mathrm{N}$ nas sessões de tutoria, sujeitar-se-á a algumas restrições em sua vida acadêmica, quais sejam: a impossibilidade de concorrer a bolsas de extensão; ter o conceito $\mathrm{N}$ como critério de desempate nas concessões de bolsas de Iniciação Científica; a impossibilidade de concorrer à bolsa de tutoria como tutor bolsista e / ou voluntário. 


\section{Impactos do TutBQI}

Para avaliação dos impactos do TutBQI no desempenho acadêmico, realizou-se um levantamento do índice de reprovação e do rendimento médio dos estudantes na BQI100, ao longo dos 10 anos de funcionamento do programa. O rendimento médio correspondeu à média aritmética das notas finais ( 0 a 100) obtidas por todos os alunos matriculados em BQI 100, no final de cada semestre. A análise foi feita comparando-se o rendimento médio entre os estudantes matriculados no TutBQI com conceito $S$, os matriculados no programa com conceito $\mathrm{N}$ e os não matriculados no TutBQI, por não atenderem aos critérios de seleção para o programa.

Apresenta-se na Figura 3 o rendimento médio dos estudantes matriculados na BQI100 ao longo de 10 anos. $\mathrm{O}$ rendimento médio dos estudantes com conceito $\mathrm{N}$ foi estatisticamente inferior ao rendimento médio obtido pelos estudantes com conceito $\mathrm{S}$, em 6 dos 10 anos, e inferior ao rendimento médio obtido pelos estudantes não-matriculados no TUTBQI, em 5 anos, com média variando entre 53,3 a 63,5. Nos demais anos, os rendimentos médios dos estudantes com conceito $\mathrm{N}$ mantiveram essa mesma tendência, embora não se tenha verificado significância estatística. Durante os 10 anos de TutBQI, o rendimento médio dos estudantes com conceito $S$ permaneceu similar ao dos estudantes não-matriculados, variando entre 66,8 a 74,4 .

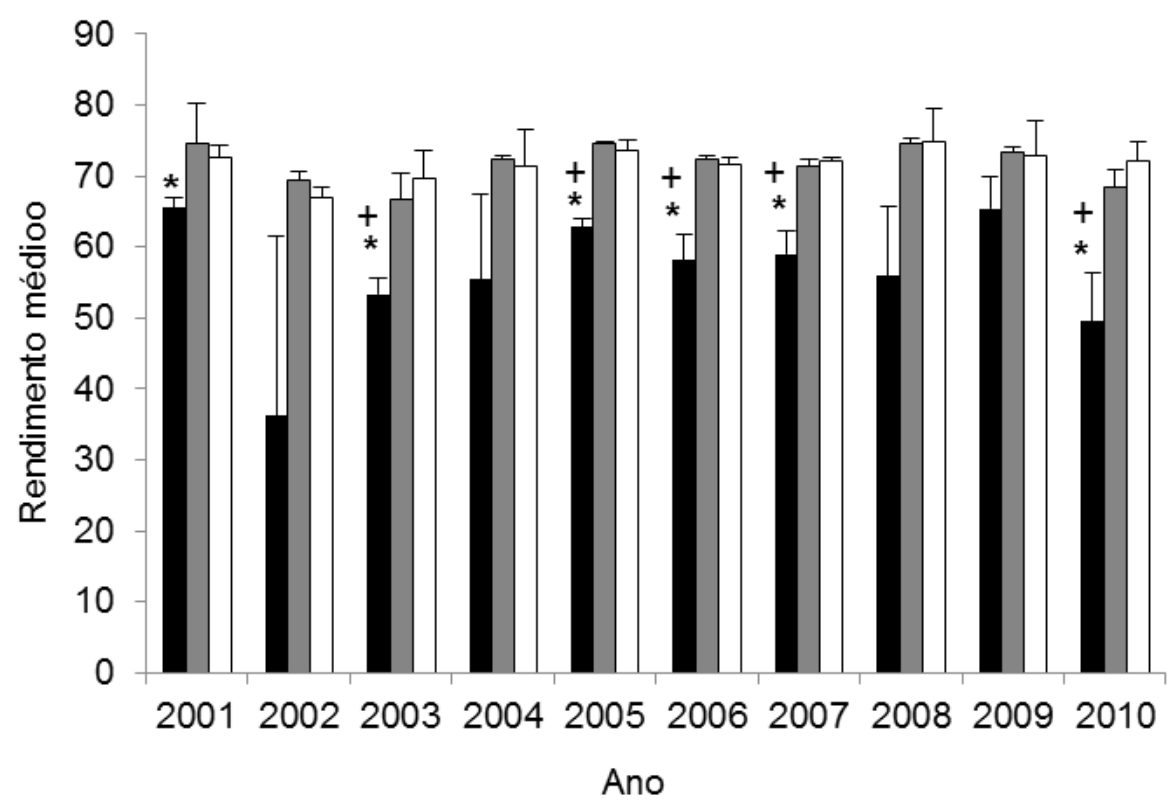

Figura 3. Rendimento médio (média das notas finais) dos estudantes matriculados na Bioquímica Fundamental (BQI100), nos anos de 2001 a 2010. $\left(^{*}\right)$ diferença estatisticamente significativa entre os rendimentos médios dos estudantes matriculados no TutBQI que obtiveram conceito $\mathrm{N}$ (barras pretas) e os que obtiveram conceito $S$ (barras brancas) e $(+)$ diferença estatisticamente significativa entre os rendimentos médios de estudantes com conceito $\mathrm{N}$ e os estudantes não matriculados (barras cinzas) no TutBQI, segundo Teste-t de Student a 5\% de significância. 
Observa-se na figura 4 que, ao longo dos 10 anos, os índices de reprovação dos estudantes com conceito $\mathrm{N}$ foram superiores aos índices de reprovação de todos os outros estudantes, com destaque para os anos de 2002 e 2003, quando mais de $40 \%$ dos estudantes com conceito $\mathrm{N}$ foram reprovados em BQI100. PPor outro lado, os índices de reprovação dos estudantes com conceito $S$, com exceção do ano de 2002, sequer atingiram $10 \%$.

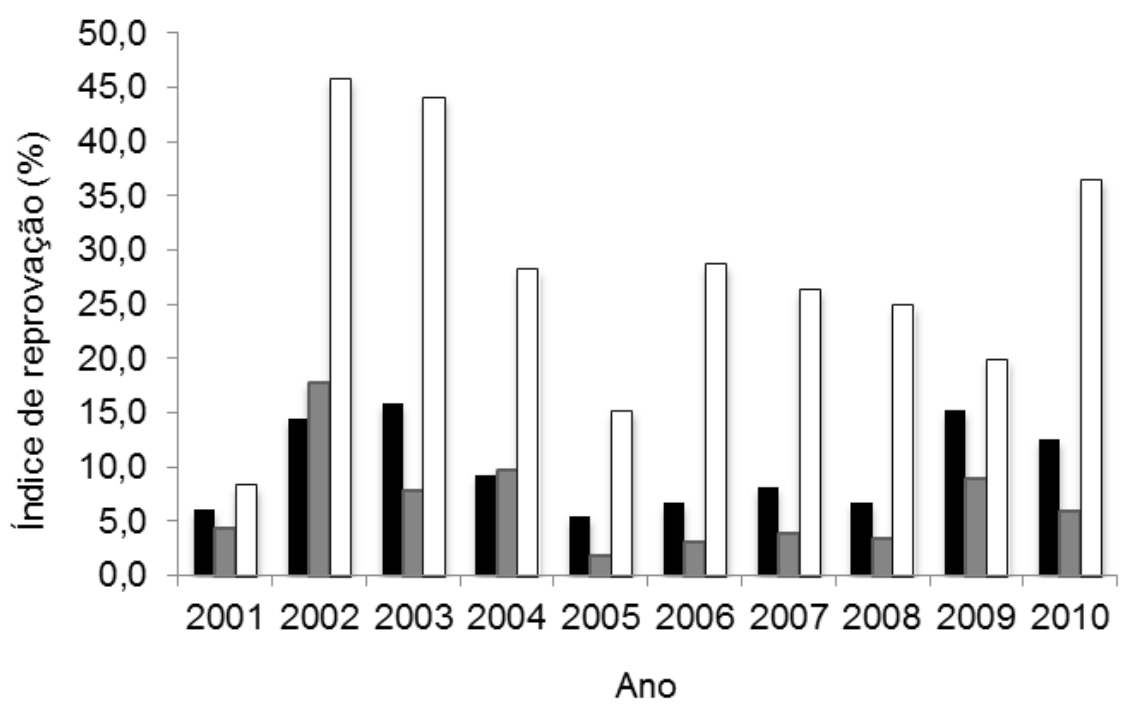

Figura 4. Índice de reprovação dos estudantes matriculados na Bioquímica Fundamental (BQI100) nos anos de 2001 a 2010. Barras pretas: estudantes não matriculados no TutBQI por não atenderem aos critérios de seleção; Barras cinzas: estudantes matriculados no TutBQI que obtiveram desempenho satisfatório (Conceito S); Barras brancas: estudantes matriculados no TutBQI que obtiveram conceito nãosatisfatório (Conceito $\mathrm{N}$ ).

Analisando esses dados, conclui-se que os estudantes que não compareciam às sessões de tutoria não conseguiram sanar suas deficiências prévias e, portanto, mantiveram um desempenho acadêmico inferior aos dos demais estudantes da BQI100. Felizmente, a grande maioria dos estudantes matriculados no TutBQI (70 a 90\%, com média de $78,7 \pm 6.8$ ) eram comprometidos com o programa e, como pôde ser percebido, alcançaram bom desempenho acadêmico, igualando-se ou até mesmo superando os estudantes não matriculados no programa, os quais, supostamente, teriam um nível de conhecimento prévio superior aos primeiros.

Questionários aplicados aos estudantes após a primeira avaliação da BQI100 revelam que os estudantes comprometidos com o programa reconhecem a importância das sessões de tutoria na motivação da aprendizagem. A alta procura pelas vagas remanescentes do TutBQI por parte de estudantes não selecionados para o programa, incluindo aqueles com desempenho satisfatório em química orgânica, é percebida pela equipe da tutoria como uma boa aceitação do programa pela comunidade estudantil. 


\section{Limitações do TutBQI}

Apesar dos impactos positivos do TutBQI, a promoção da efetiva aprendizagem, como um processo de construção do conhecimento, desenvolvimento de visão crítica e aquisição de habilidade para integrar ideias e solucionar problemas, permanece um desafio.

Quando o TutBQI foi criado, esperava-se que as sessões fossem conduzidas de forma a reforçar conceitos abordados nas aulas expositivas através da participação dinâmica dos estudantes em discussões e resoluções de exercícios. Essa proposta se aproximaria dos métodos originais de ensino em pequenos grupos, nos quais os estudantes participam ativamente da sua aprendizagem e são capacitados para a aplicação de conceitos aprendidos [5,6,7]. No entanto, a baixa assimilação dos conceitos previamente vistos em sala de aula e a inibição dos estudantes têm dificultado a dinâmica inicialmente pensada. Embora os tutores procurem encorajar a participação ativa dos estudantes na formulação de respostas para seus questionamentos e na resolução dos exercícios propostos, as sessões de tutoria têm sido conduzidas em um formato que mais caracteriza uma mini-aula expositiva.

Os tutores frequentemente lidam com estudantes desmotivados que admitem sua falta de atenção ou infrequência nas aulas da BQ100. Alguns aspectos possivelmente contribuem para esse comportamento: o amplo e complexo conteúdo que deve ser ministrado em 180 horas distribuídos ao longo de 15 semanas; a heterogeneidade dos estudantes matriculados em uma mesma classe, os quais são provenientes de diferentes programas de graduação e possuem diferentes níveis de conhecimento prévio; a imaturidade dos estudantes para compreender a importância da disciplina na sua formação acadêmica; finalmente, a pobre experiência dos professores em métodos alternativos de ensino.

Outro desafio a ser superado é a resistência inicial da alguns estudantes ao TutBQI que veem o programa como uma atividade extra classe a ser conciliada com outras disciplinas regulares. Estudantes desinteressados param de frequentar as sessões de tutoria antes mesmo da primeira avaliação da BQI100. No entanto, é justamente após a primeira avaliação que os estudantes que permanecem no programa passam a valorizar as sessões de tutoria devido às boas notas obtidas.

Uma sugestão para se tentar reverter o cenário relatado acima seria a inovação das aulas expositivas por meio da incorporação de atividades que estimulem a ativa participação dos estudantes e que proporcione feedback imediato para os professores. 
Um exemplo de ferramenta que tem sido utilizado em disciplinas com grandes turmas, apresentando resultados positivos no engajamento de alunos e na melhora da aprendizagem, é o método Audience Response System (ARS) ou clicker [8,9,10,11]. Os ARS correspondem a hardwares e softwares que permitem estudantes responderem questões mostradas em uma tela, utilizando um dispositivo de controle remoto [12]. Após os alunos responderem uma votação, os resultados são apresentados para a classe em um formato visual, usualmente um histograma [13]. Dessa forma, os ARS proporcionam feedback contínuo para os estudantes e para o professor sobre o desempenho da classe.

Adequando essa ou outras ideias à realidade brasileira, tornar-se-ia possível a criação de uma atmosfera propícia à aprendizagem dinâmica. A consequência poderia ser positiva na TutBQI no sentido de se reduzir o tempo gasto pelo tutor na revisão das informações previamente apresentadas na BQI100 e de se criar oportunidade para uma verdadeira discussão de situações cotidianas relacionadas à Bioquímica. Os estudantes ocupariam finalmente a posição central no seu processo de aprendizagem como um sujeito que escuta, pensa, questiona, discute e constrói conhecimento.

Adicionalmente, no intuito de se encorajar o comprometimento dos estudantes matriculados no TutBQI, seria interessante reforçar a divulgação na comunidade acadêmica dos impactos positivos do programa sobre o desempenho dos estudantes.

\section{Referências}

[1] Instituto Nacional de Estudos e Pesquisas Educacionais Anísio Teixeira (Inep), 2011. http://portal.inep.gov.br/web/censo-da-educacao-superior/evolucao-1980-a-2007 Acesso em 20 de maio de 2013.

[2] Passos FJV, Braathen PC, Guerreiro M, Arruda MA, Bohnenberger JC. Programa de Tutoria: Uma experiência. In: XXIX Congresso Brasileiro de Ensino de Engenharia; 2001, dez 19-22; Porto Alegre, Brasil. Brasília: Associação Brasileira de Ensino de Engenharia, 2001. p. 63-68.

[3] Pereira MCB, Mendes FQ, Sartori MA, Dias AS, Pena LJ, Moura, VZV, Capucho, AS. Tutoria em Bioquímica: biomoléculas. Viçosa: UFV, 2008. 50 p.

[4] Pereira MCB, Mendes FQ, Sartori MA, Dias AS, Pena, LJ, Moura VZV, Capucho AS. Tutoria em Bioquímica: metabolismo celular. Viçosa: UFV, 2006. 74 p.

[5] Mistry C. Student participation in the form of assessed tutorials. Biochem Educ 1994; 22: $11-12$.

[6] Wood EJ. Tutorials and small group teaching. Biochem Educ 1998; 16: 13-16.

[7] Anderson WL, Mitchell SM, Osgood MP. Comparison of student performance incooperative learning and traditional lecture-based biochemistry classes. Biochem Mol 
Biol Educ 2005; 33: 387-393.

[8] Herreid CF. "Clicker" cases: introducing case study teaching into large classrooms. J Coll Sci Teach 2006; 36, 43-47.

[9] Caldwell JE. Clickers in the large classroom: current research and best-practice tips. CBE-LSE 2007: 6, 9-20.

[10] Gauci SA, Dantas AM, Williams DA, Kemm RE. Promoting student-centered active learning in lectures with a personal response system. Adv Physiol Educ 2009; 33, 60-71.

[11] Cain J, Black EP, Rohr J. An Audience Response System strategy to improve student motivation, attention, and feedback. Am J Pharm Educ 2009; 73: 1-7.

[12] Kay $\mathrm{RH}$, Lesage A. Examining the benefits and challenges of using audience response systems: A review of the literature, Aust J Educ Tech 2009; 53 (3): 819-827.

[13] Mantoro T, Ayu MA, Habul E, Khasanah AU. Survnvote: A Free Web Based Audience Response System to Support Interactivity in the Classroom. In: IEEE Conference on Open System; 2010, dec 5-7; Kuala Lumpur, Malaysia. p.34-39.

\section{Agracecimentos}

Bruna Moraes agradece a CAPES pela concessão de bolsa de doutorado pelo programa REUNI. Tutores agradecem à Pró-reitoria de Ensino pela concessão de suas bolsas. Os autores agradecem a todos os professores que já contribuíram para o TutBQI e à secretaria do programa pela coleta dos dados apresentados neste artigo. Agradecimentos aos Professores Vicente de Paula Lelis e Frederico Passos pelas sugestões para melhoria deste artigo. 